\title{
Proving Bounds for Real Linear Programs in Isabelle/HOL
}

\author{
(Extended Abstract)
}

\author{
Steven Obua ${ }^{\star}$ \\ Technische Universität München \\ D-85748 Garching, Boltzmannstr. 3, Germany \\ e-mail: obua@in.tum.de, url: http://www4.in.tum.de/ obua
}

The Flyspeck project [3] has as its goal the complete formalization of Hales' proof [2] of the Kepler conjecture. The formalization has to be carried out within a mechanical theorem prover. For our work described in this paper, we have chosen the generic proof assistant Isabelle, tailored to Higher-Order Logic (HOL) [4]. In the following, we will refer to this environment as Isabelle/HOL.

An important step in Hales' proof is the maximization of about $10^{5}$ real linear programs. The size of these linear programs (LPs) varies, the largest among them consist of about 2000 inequalities in about 200 variables. The considered LPs have the important property that there exist a priori bounds on the range of the variables. The situation is further simplified by our attitude towards the linear programs: we only want to know wether the objective function of a given LP is bounded from above by a given constant $K$.

Under these assumptions, Hales describes [1] a method for obtaining an arbitrarily precise upper bound for the maximum value of the objective function of an LP. This method still works nicely in the context of mechanical theorem provers. The burden of calculating the upper bound is delegated to an LP solver that needs not to be trusted. Instead, the LP solver delivers a small certificate to Isabelle/HOL that can be checked cheaply. Furthermore, there is no need to delve into the details of the actual method of optimizing an LP, which is usually the Simplex method. These details just do not matter for the theorem prover.

The method can be described conveniently using matrices. In order to reason with and about matrices in Isabelle/HOL, we have invented the notion of finite matrices, and use finite matrices to represent linear programs. Finite matrices can be fitted into the system of numeric axiomatic type classes in Isabelle/HOL via the algebraic concept of lattice-ordered rings $[8,9]$. The hierarchy of type classes in Isabelle/HOL that organize numerical theories [5] had to be changed for this.

Checking the certificate from the external LP solver is basically a calculation involving finite matrices, and the matrices we have to deal with coming from our Flyspeck background are sparse, therefore we present a sparse matrix representation of finite matrices and formalize operations like sparse matrix multiplication.

\footnotetext{
* Supported by the Ph.D. program "Logik in der Informatik" of the "Deutsche Forschungsgemeinschaft."
}

Dagstuhl Seminar Proceedings 05021 
Using a rewriting oracle that is based on the ideas found in [7], these operations can be performed so quickly that it is projected that the linear programs arising in the proof of the Kepler conjecture can be bounded in about 10 days on a 3Ghz Pentium 4.

\section{References}

1. Thomas C. Hales. Some algorithms arising in the proof of the Kepler conjecture, sect. 3.1.1., arXiv:math.MG/0205209

2. Thomas C. Hales. A Proof of the Kepler Conjecture, Annals of Mathematics, to appear.

3. The Flyspeck Project Fact Sheet. http://www.math.pitt.edu/ thales/flyspeck/index.html

4. Tobias Nipkow, Lawrence C. Paulson, Markus Wenzel. Isabelle/HOL: A Proof Assistant for Higher-Order Logic, Springer 2002

5. Lawrence C. Paulson. Organizing Numerical Theories Using Axiomatic Type Classes. Journal of Automated Reasoning, in press.

6. Two Fast Algorithms for Sparse Matrices: Multiplication and Permuted Transposition. ACM Transactions on Mathematical Software, Vol 4. No 3, pp. 250-269, 1978.

7. Bruno Barras. Programming and Computing in HOL. TPHOLs 2000, LNCS 1869, pp. 17-37, Springer 2000.

8. Garrett Birkhoff. Lattice Theory, AMS 1967.

9. László Fuchs. Partially ordered algebraic systems, Addison-Wesley 1963. 